\title{
Influence of operating time on the corrosion in single-phase and two-phase media
}

\author{
Valery I. Baranenko ${ }^{1}$, Olga M. Gulina ${ }^{2}$, Nikolay L. Salnikov ${ }^{3}$ \\ 1 All-Russian Research Institute for Nuclear Power Plant Operation (JSC VNIIAES), 25 Ferganskaya St., 109507 Moscow, Russia \\ 2 Joint Stock Company OKB "Gidropress", 21 Ordzhonikidze St., 142103 Podolsk, Moscow Reg., Russia \\ 3 Obninsk Institute for Nuclear Power Engineering, NRNU MEPhI, 1 Studgorodok, 249040 Obninsk, Kaluga Reg., Russia \\ Corresponding author: Olga M. Gulina (omgulina18@mail.ru)
}

Academic editor: Yury Korovin • Received 14 October 2020 • Accepted 4 April 2021 • Published 23 June 2021

Citation: Baranenko VI, Gulina OM, Salnikov NL (2021) Influence of operating time on the corrosion in single-phase and two-phase media. Nuclear Energy and Technology 7(2): 127-132. https://doi.org/10.3897/nucet.7.69175

\begin{abstract}
Modern foreign computer codes predict a linear growth in the pipeline wall thinning with time due to the process of flow-accelerated corrosion (FAC), i.e. erosion-corrosion wear (ECW). Linear time-thinning dependence and corrosion rate constancy are not however typical of the NPP piping operating conditions. And the associated excessive conservatism of the residual life estimates leads to increased economic costs of repeated inspections. In domestic software tools, EKI-02 and EKI-03, the influence of operating time are taken into account by introducing the respective coefficient into the Chexal-Horowitz model based on the yield of corrosion products into the coolant. The ECW intensity can be however reduced through improvements in operating conditions, preventive measures, improvements in water chemistry, etc., and the use of the dependences once obtained may turn out to be too conservative. Based on a large number of repeated measurements as well as on data from corrosion testers, it has been shown that the influence of time can be described by the function of a particular form, the coefficients of which differ for different units and component and subsystem types. This makes it possible to determine the 'aging function' based on inspection data, and then use it in a targeted way for particular components. It has been shown that such estimates are much less conservative.
\end{abstract}

\section{Keywords}

Erosion-corrosion wear, corrosion rate estimation, pipeline wall thinning, Chexal-Horowitz Flow-Accelerated Corrosion Modelt

\section{Introduction}

Erosion-corrosion wear (ECW) is a widespread type of damage to the NPP thermomechanical equipment and pipelines. Practically all components of the turbine plant condensate feed and steam lines at NPPs and heat power stations are exposed to ECW. Other types of metal wear occur in most cases in parallel with this process (Henzel and Egan 1989, Chexal and Horowitz 1995, Poulson 1999, Dooley and Chexal 2000, Horowitz and Smith 2013).

Early versions of the codes for the ECW calculation were developed at the USA's Electric Power Research Institute (EPRI). In the 1980s and 1990s, CHEC, CHECNDE, CHEC-T, CHECMATE, CHECWORKS, and other codes were developed. It was assumed in the process

* Russian text published: Izvestiya vuzov. Yadernaya Energetika (ISSN 0204-3327), 2021, n. 1, pp. $29-40$. 
of the code development that the thinning values were directly proportional to time (Kain et al. 2008, Zander 2012). Such dependence of the thinning value on time is valid if conditions do not change during operation and the secondary processes involved in the formation of protective films play no role (Akimov 1946), that is, if the factors which accelerate and slow down corrosion are approximately balanced by each other.

A linear dependence of the thinning value on time and the constancy of the corrosion rate are not typical of the NPP pipeline operating conditions. At the same time, excessively conservative estimates lead to increased economic costs of repeated inspections, since, in this case, the predicted residual life is much shorter than with the decreasing ECW rate. Such approach to thinning estimates is naturally highly conservative. The question of whether time shall or shall not be taken into account remains open.
The purpose of the study is to investigate the problem based on the wall thickness inspection data, including the analysis of repeated measurements, and the data on the damaged pipelines, as well as to analyze the corrosion tester readings.

\section{ECW rate prediction model}

The analog for Russian software tools, EKI-02 and EKI-03, (the authors of this paper are the developers of these codes) is the CHECWORKS (Chexal-Horowitz Engineering Corrosion Workstation) code (ASME code Case N-480). The codes were developed using foreign experience and the results of domestic investigations (Gulina et al. 2010, Baranenko et al. 2012, Gulina and Frolova 2012, Naftal et al. 2014). The ECW and wall thinning rates are calculated using the EKI02 and EKI-03 codes by the following equations:

$$
W_{\mathrm{ECW}}=C_{0} \times F_{1}(T) \times F_{2}(X C) \times F_{3}(\mathrm{v}) \times F_{4}\left(\mathrm{O}_{2}\right) \times F_{5}(\mathrm{pH}) \times F_{6}\left(K_{k}\right) \times F_{7}(\alpha) \times F_{8}(A) \times F_{9}(\tau),
$$

$$
\Delta S(\tau)=\int_{\tau 0}^{\tau} W_{E C W} d \tau,
$$

where $W_{\mathrm{ECW}}$ is the corrosion rate, $\mathrm{mm} /$ year; $C_{0}$ is the coefficient equal to $1 \mathrm{~mm} /$ year; $F_{1}(T)$ is the coefficient that corrects for temperature; $F_{2}(X C)$ is the coefficient that corrects for the metal composition; $F_{3}(v)$ is the coefficient defined by the medium rate; $F_{4}\left(\mathrm{O}_{2}\right)$ is the coefficient that corrects for the oxygen concentration; $F_{5}(\mathrm{pH})$ is the coefficient that corrects for the $\mathrm{pH}$ value; $F_{6}\left(K_{k}\right)$ is the coefficient that corrects for the pipeline geometry (Keller coefficient); $F_{7}(\alpha)$ is the coefficient that corrects for steam humidity (for a single-phase medium, $F_{7}(\alpha)=1$ ); $F_{8}(A)$ is the coefficient that corrects for the amine used (ammonia, morpholine, ethanolamine); and $F_{9}(\tau)$ is the coefficient that corrects for the component operating time; $\Delta S(\tau)-$ wall thinning for the estimated time interval $\left(\tau_{0}, \tau\right)$.

A parameter has been introduced into the EKI-02 and EKI-03 codes to take into account the influence of operating time on the corrosion rate and the wall thinning value which allows estimating the ECW and thinning rate while staying within the 'reasonable conservatism' limits.

The time parameter introduced into the EKI-02 and EKI-03 codes allows one to calculate

- the ECW rate at the pipeline operation start time;

- the ECW rate at the pipeline operation end time;

- the average ECW rate for the estimated time interval;

- the pipeline wall thinning for the estimated time interval.

It was assumed in determining the dependence which describes the influence of operating time on corrosion rate, that the concentration of iron in feedwater depends on the escape of iron in the process of the secondary circuit equipment and piping ECW.
The content of iron reflects just indirectly the ECW processes, since there are more factors such as contribution of downtime corrosion to the corrosion processes, measures to remove corrosion products from equipment, and others. Naturally, the model simplification at the expense of such factors affects the code calculation accuracy. Currently, there is a sufficient array of data accumulated on the intensity of the ECW process in the pipeline components at NPPs with different reactor facilities. Analyzing such data makes it possible to propose a methodology to estimate the individual dependence of the ECW rate on time and to use it to predict the thinning of the pipeline component and to calculate its residual life.

\section{Estimation of the ECW rate for components of pipelines with single- and two-phase media based on reinspection data (VVER-440 NPPs)}

The corrosion rate is estimated based on inspection data as follows (Baranenko et al. 2016, 2017):

$$
W_{\mathrm{ecw}}=\left(S_{\mathrm{nom}}-S_{\mathrm{min}}\right) / \tau \text {, }
$$

where $W_{\text {ecw }}$ is the ECW rate based on in-service inspection data, $\mathrm{mm} /$ year; $S_{\text {nom }}$ is the nominal wall thickness value, $\mathrm{mm} ; S_{\min }$ is the minimum wall thickness value based on in-service inspection data, $\mathrm{mm}$; and $\tau$ is the time between the component operation start and the inspection date.

In the process of follow-up measurements, pointwise estimates of the ECW rate, obtained using formula (3), are approximated by the function of the form $f(\tau)=a \tau^{-b}$. 
The corrosion rate calculation results for feedwater pipelines (FWP) and steam lines (SP) are presented in Figs 1, 2 and in Tables 1, 2 where $W_{\text {ecw }}$ is pointwise estimates, and $W_{\text {trend }}$ is their approximation.

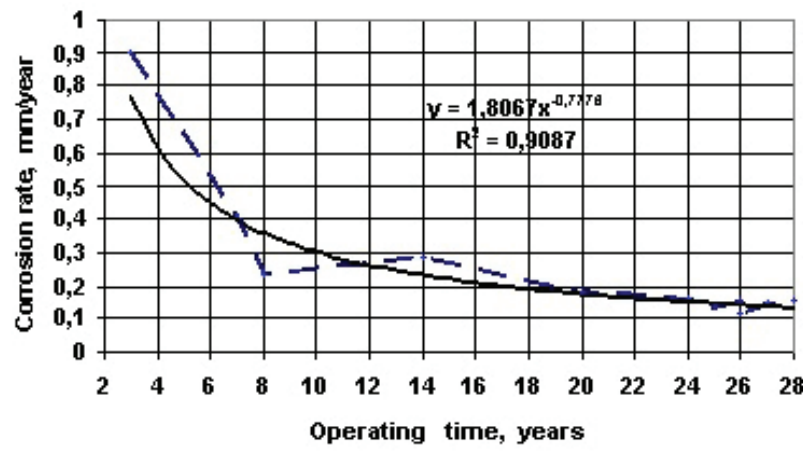

Figure 1. ECW rate for the VVER-440 NPP feedwater pipelines.

Table 1. Feedwater pipeline ECW rate obtained from inspection data and its approximation

\begin{tabular}{lcccc}
\hline No. & Date, year & $\boldsymbol{\tau}$, years & $\boldsymbol{W}_{\text {ecw }}, \mathbf{m m} /$ year & $\boldsymbol{W}_{\text {trend }}$ fw, $\mathbf{m m} /$ year \\
\hline 1 & 1984 & 3 & 0.900 & 0.772 \\
2 & 1988 & 7 & 0.400 & 0.396 \\
3 & 1989 & 8 & 0.238 & 0.357 \\
4 & 1995 & 14 & 0.286 & 0.230 \\
5 & 2001 & 20 & 0.180 & 0.174 \\
6 & 2001 & 20 & 0.185 & 0.174 \\
7 & 2005 & 24 & 0.158 & 0.146 \\
8 & 2006 & 25 & 0.136 & 0.141 \\
9 & 2007 & 26 & 0.154 & 0.142 \\
10 & 2007 & 26 & 0.112 & 0.142 \\
11 & 2008 & 27 & 0.148 & 0.138 \\
12 & 2009 & 28 & 0.154 & 0.134 \\
\hline
\end{tabular}

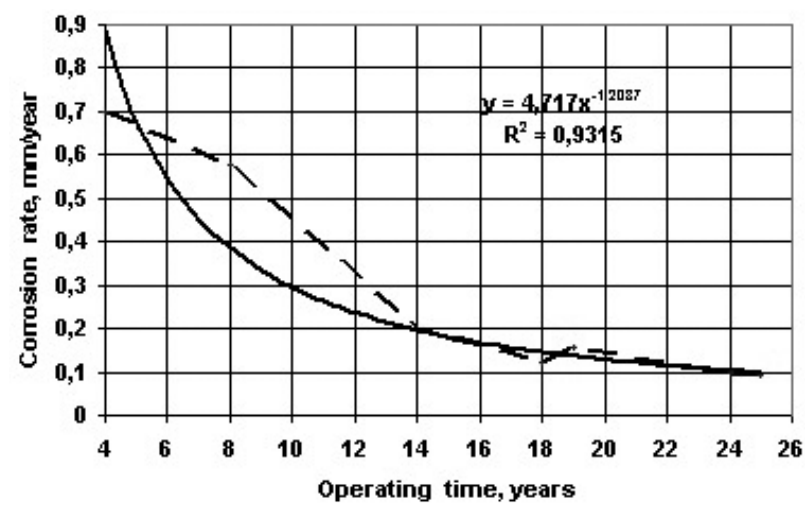

Figure 2. ECW rate for the VVER-440 NPP main steam line.

For feedwater, the dependence for the ECW rate is

$$
W_{\text {trend }}{ }^{\mathrm{fw}}=1.725 \cdot \tau^{-0.75} \text {, }
$$

and for steam lines, it is

$$
W_{\text {trend }}{ }^{\mathrm{sl}}=3.307 \cdot \tau^{-1.10} \text {. }
$$

The following dependence is used to determine the pipeline operating time, $\Delta \tau$, until the minimum allowable thickness (residual life) is reached
Table 2. Steam line ECW rate obtained from inspection data and its approximation

\begin{tabular}{lcccc}
\hline No. & Date, year & $\boldsymbol{\tau}$, years & $\boldsymbol{W}_{\text {ecw }}, \mathbf{m m} / \mathbf{y e a r}$ & $\boldsymbol{W}_{\text {trend }}$ sl, $\mathbf{m m} / \mathbf{y e a r}$ \\
\hline 1 & 1985 & 4 & 0.700 & 0.882 \\
2 & 1989 & 8 & 0.575 & 0.381 \\
3 & 1995 & 14 & 0.200 & 0.194 \\
4 & 1999 & 18 & 0.120 & 0.143 \\
5 & 2000 & 19 & 0.158 & 0.134 \\
6 & 2005 & 24 & 0.096 & 0.101 \\
7 & 2006 & 25 & 0.092 & 0.096 \\
\hline
\end{tabular}

$$
\Delta \tau=\left(S_{\min }-S_{\text {all }}\right) / W_{\text {ecw }},
$$

where $\Delta \tau$ is the residual life; and $S_{\text {all }}$ is the minimum allowable thickness value, $\mathrm{mm}$.

The smooth lines in Figs 1, 2 show the trend approximation.

Fig. 3 presents the steam line ECW rates calculated with the use of codes and obtained based on the inspection data trend. It has been shown that both curves agree well and it is possible to use them to predict the ECW rate: after 20 to 25 years of operation, the rate values are practically identical and are close to a constant.

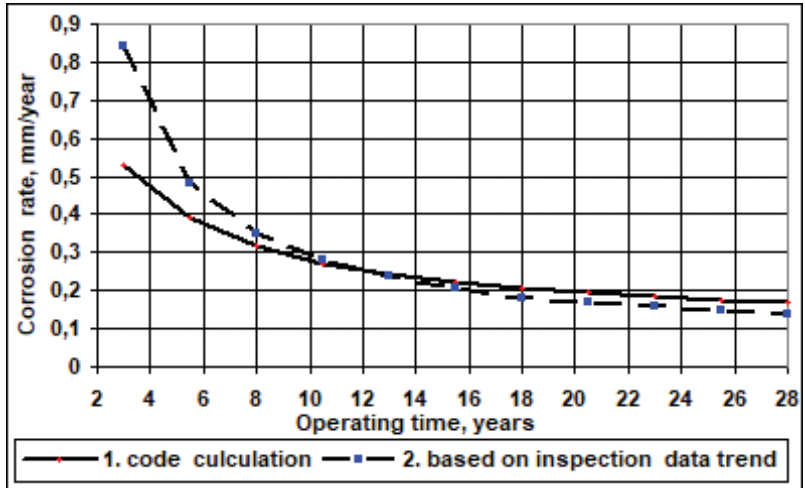

Figure 3. ECW rate for the VVER-440 NPP main steam line: 1 - code calculation; 2 - based on inspection data trend.

\section{Time analysis of corrosion tester readings for VVER-440 NPPs}

Corrosion testers (CT) are used to calculate the metal corrosion rate (corrosion penetration depth) for different components and pipelines. Testers compute the corrosion penetration rate value as the ratio of the loss of weight to the metal surface area and density. In other words, this is a characteristic of the metal general corrosion in a medium.

To determine the nature of the CT corrosion wear at VVER-440 NPPs, there were processed the corrosion penetration rate values for $27 \mathrm{CTs}$ installed on components with a single-phase medium in three VVER-440 NPP units with the exposure time between 7.9 to 61.3 thousand hours (Table 3 ). In all units, the corrosion penetration rate $W_{\text {pen }}$ changed practically equally, from 5 to 0.6 $\mu \mathrm{m} /$ year, which made it possible to merge the statistics for all units to obtain the averaged result. 
Table 3. Corrosion penetration rate for CTs at VVER-440 NPPs

\begin{tabular}{lccccc}
\hline No. & Exposure, $\mathbf{h}$ & $\boldsymbol{W}_{\text {pen }}, \boldsymbol{\mu m} / \mathbf{y e a r}$ & No. & Exposure, $\mathbf{h}$ & $\boldsymbol{W}_{\text {pen }}, \boldsymbol{\mu m} / \mathbf{y e a r}$ \\
\hline 1 & 7896 & 5 & 14 & 18456 & 2 \\
2 & 8568 & 3 & 15 & 19128 & 1.2 \\
3 & 8736 & 4 & 16 & 24960 & 2 \\
4 & 8784 & 4 & 17 & 25848 & 0.6 \\
5 & 8856 & 3 & 18 & 26304 & 3 \\
6 & 8928 & 4.1 & 19 & 33168 & 1.5 \\
7 & 9528 & 3 & 20 & 35208 & 2.5 \\
8 & 10224 & 3.2 & 21 & 35808 & 2 \\
9 & 17208 & 2 & 22 & 41472 & 1.1 \\
10 & 17640 & 2 & 23 & 43416 & 1.1 \\
11 & 17784 & 3 & 24 & 51624 & 0.97 \\
12 & 17904 & 2 & 25 & 51672 & 0.6 \\
13 & 18336 & 1 & 26 & 61224 & 0.9 \\
& & & 27 & 61296 & 0.85 \\
\hline
\end{tabular}

Fig. 4 presents the values of the corrosion penetration rate, $W_{\text {pen }}$, and shows the trend line and its equation in the form of a power dependence.

It should be noted that processing and interpretation of inspection data are one of the most complex issues (Baranenko et al. 2017, 2020). It can be seen when comparing the ECW rates in the FW pipeline ( 0.900 to 0.112 $\mathrm{mm} /$ year) and in corrosion tasters in a single-phase medium ( 5 to $0.6 \mu \mathrm{m} /$ year) that the intensity of general corrosion (testers) is two orders of magnitude as low as that determined based on the pipeline inspection data. And the time change is described by a function of the same form. One of the explanations to this fact is that the ECW rate is estimated from the maximum thinning as recorded in the course of inspections (local effect). For corrosion testers, however, the penetration rate was calculated as the surface average value that characterizes general corrosion. The pipeline wall thickness value, average for all measurements, also differs very slightly from the nominal thickness (a few percent), since the pipeline life is defined largely by local effects (flow turbulence, weld adjacent zones, metal composition in particular areas, etc.) and not by general corrosion in the pipeline.

\section{Time analysis of corrosion tester readings for RBMK-1000 NPPs}

The corrosion penetration rate was calculated for CTs of carbon and high-chromium steels installed in pipelines at RBMK-1000 NPP units. While carbon-steel CTs are subject to corrosive wear during operation in an aqueous medium, high-chromium steels and alloys corrode electrochemically which is defined by the presence of the electrode potential.

For steel 20, the exposure time in different pipelines at NPP 1 and NPP 2 is from 10 to 113 thousand hours. The corrosion penetration rate values lie in a range of 1 to $11.5 \mu \mathrm{m} /$ year.

In a general form, the equations to calculate the corrosion penetration rate $W_{\text {pen }}$ are reduced to the form

$$
W_{\text {pen }}=C_{\mathrm{o}} \times \tau^{-n}, \mu \mathrm{m} / \text { year, }
$$

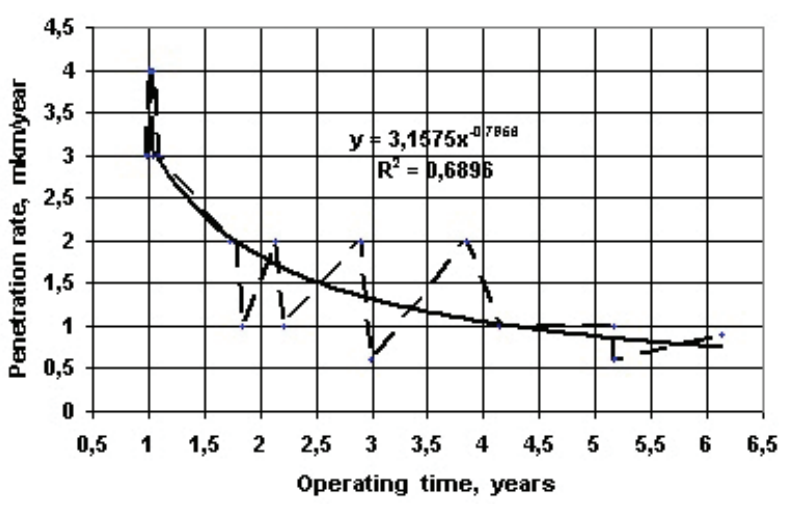

Figure 4. Corrosion penetration rate on CTs for components with a single-phase medium at VVER-440 NPPs $\left(\mathrm{W}_{\text {pen }}=\right.$ $\left.3.1575 \times \tau^{-0.7956}\right)$.

where $C_{\mathrm{o}}$ is the coefficient; $\tau$ is the CT exposure time, years; and $n$ is the power index.

The average error of the corrosion penetration rare calculation, according to the trend with respect to the inspection data, does not exceed $10 \%$.

The $W_{\text {pen }}$ diagram for the saturated steam pipeline CTs is presented in Fig. 5 showing the trend line and the approximation certainty value.

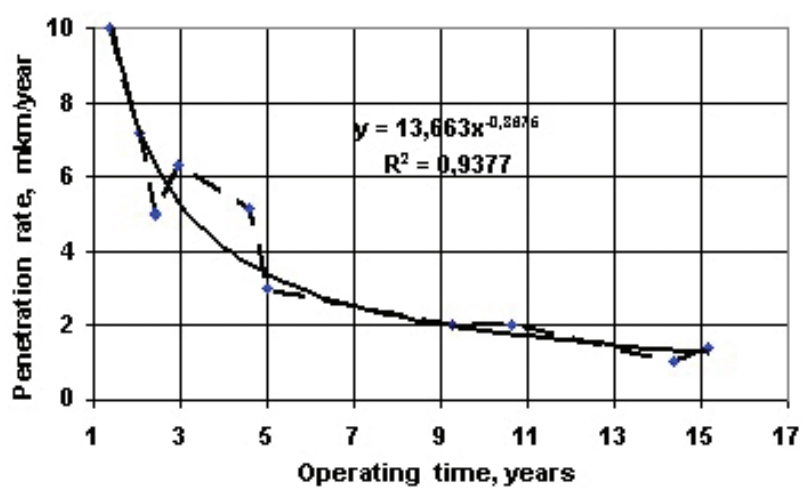

Figure 5. Corrosion penetration rate for a saturated steam pipeline.

Fig. 6 shows processed data from CTs of the 08Cr18N10T austenitic steel in an RBMK-1000 NPP's FW pipeline.

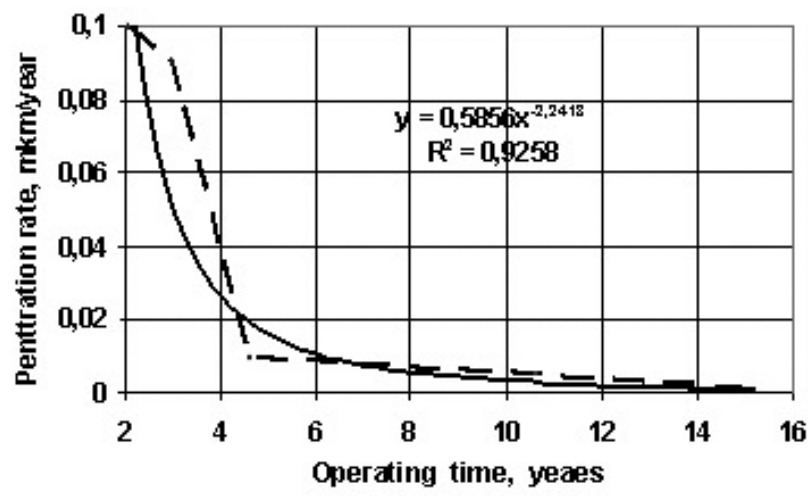

Figure 6. Corrosion penetration rate for the RBMK-1000 NPP feedwater pipeline (08Cr18N10T austenitic steel). 
A similar function describes the dependence of the corrosion penetration rate for $\mathrm{CTs}$ in saturated steam pipelines.

$$
W_{\text {pen }}^{\text {ss }}=0.4126 \times \tau^{-0.921}
$$

Data from corrosion testers in stainless-steel pipelines at NPP 2, also with the RBMK-1000 reactor, is presented in Table 4 and in Fig. 7.

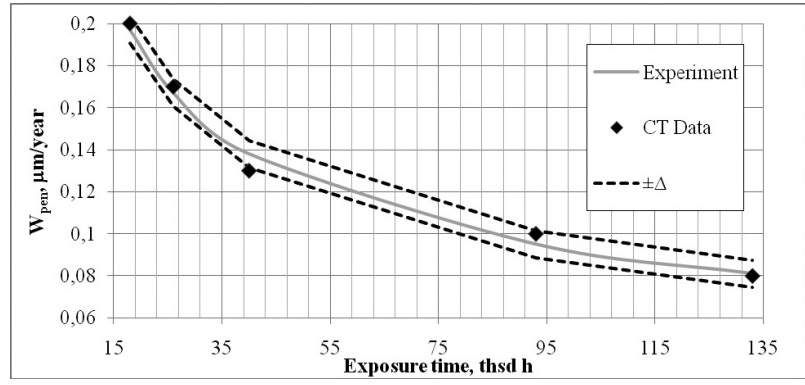

Figure 7. Confidence intervals for the stainless-steel CT corrosion penetration rate (approximation $W_{\text {pen }}=0.7052 \times \tau^{-0.4415}$ ), $\mu \mathrm{m} / \mathrm{year}$

Table 4. Corrosion penetration rate in $08 \mathrm{Cr} 18 \mathrm{~N} 10 \mathrm{~T}$ steel

\begin{tabular}{lccccc}
\hline Exposure time, thsd $\mathrm{h}$ & 18 & 26 & 40 & 93 & 133 \\
$W_{\text {pen }}, \mu \mathrm{m} /$ year & 0.20 & 0.17 & 0.13 & 0.10 & 0.08 \\
\hline
\end{tabular}

Calculation of confidence intervals for the trend:

$$
\Delta_{s, t}=t_{n, \beta} \cdot\left(S^{2} / n\right)^{1 / 2}
$$

where $\Delta_{s, t}$ is the confidence interval value; $\beta$ is the confidence probability $(\beta=0.95) ; n$ is the number of measurements ( sample size $=5$ ); $t_{n, \beta}$ is Student's inverted distribution (for $n=5$ and $\beta=0.95$, the value $t_{n, \beta}=2.776$ ); and $S^{2}$ is the sampling variance:

$$
S=\sqrt{\sum_{i=1}^{n}\left(f\left(t_{i}\right)-y_{i}\right)^{2} /(n-1)}
$$

where $f\left(t_{i}\right)$ is the rate value according to the trend at the time $t_{i} ; y_{i}$ is the rate values according to the experimental data at the time $t_{i}$; and $t_{i}$ is the exposure time. Hence, we get the following results: $S=0.0052 ; \Delta_{s, t}=0.0064$ (Fig. 7).

\section{References}

- Akimov GV (1946) Fundamentals of the Theory of Corrosion and Protection of Metals. Moscow. Fizmatgiz Publ., 461 pp. [in Russian]

- ASME code Case N-480 () Examination Requirements for Pipe Wall Thinning Due to Single Phase Erosion and Corrosion. Section XI, Division: 787-795.

- Baranenko VI, Gulina OM, Mironov SA, Salnikov NL (2020) Repeated measurements and quality of estimates in the analysis of the NPP piping erosion-corrosion wear. Izvestiya vuzov. Yadernaya energetika 3: 17-29. https://doi.org/10.26583/npe.2020.3.02 [in Russian]
In nuclear power, the corrosive impacts of the medium on the material of structural components in conditions of operation are taken into account using allowance $C_{2}$ which characterizes the influence of the medium on the structural material in operating conditions (Kain et al. 2008, Zander 2012). Allowance $C_{2}$ for corrosion-resistant austenitic alloys and high-chromium steels is assumed to be equal to $0.1 \mathrm{~m}$ for an operating time of 30 years which corresponds to the general corrosion rate equal to $3.3 \mu \mathrm{m} /$ year. This value exceeds the corrosion penetration rate for stainless steels obtained from the CT data processing by about an order of magnitude.

The greatest value of the corrosion penetration depth for an exposure time of 60 years, predicted from the obtained functional dependences, is equal to $2.64 \mu \mathrm{m}$, that is, much below $0.1 \mathrm{~mm}$.

\section{Conclusion}

The experience of operation, repeated measurements, and an analysis of corrosion tester data show a substantial reduction in the ECW rate with time. Therefore, ignoring the effects of time on the ECW rate estimation leads to excessive conservatism in estimating the residual life.

Apart from the time function built in the EKI-02 and EKI-03 codes, one can use the dependence obtained from repeated measurements on components of a particular NPP. It has been shown that this dependence for different pipelines is the same: $f(\tau)=a \tau^{-b}$.

It has been shown that, for a time span of up to 20 to 25 years, the time function used in the EKI-02 and EKI-03 codes leads to more conservative residual life values than the functions obtained based on inspection results; the difference is nullified with longer operating times.

The processing of data for corrosion testers in one- and two-phase media has shown the same dependence for the corrosion penetration rate as for pipelines. However, unlike the ECW rate (local effect), these values characterize general corrosion.

Using the functional dependences obtained based on inspection data makes it possible to reduce the conservatism in calculating the ECW and general corrosion rate during the specified life period and to predict adequately the values of these characteristics for the extended life period.

Baranenko VI, Gulina OM, Salnikov NL (2017) Corrosion rate and residual life calculation for the NPP piping components based on inspection data. Izvestiya vuzov. Yadernaya energetika 4: 83-94. https://doi.org/10.26583/npe.2017.4.08 [in Russian]

- Baranenko VI, Gulina OM, Salnikov NL, Murzina OE (2016) Justification of the erosion-corrosion wear rate and service life calculations for the NPP pipelines based on in-service inspection data. Izvestiya vuzov. Yadernaya energetika 2: 55-65. https://doi.org/10.26583/ npe.2016.2.06 [in Russian] 
- Baranenko VI, Prosvirnov AA, Yevropin SV, Arefyev AA, Yurmanov VA, Gulina OM (2012) Development of software tools and regulatory documents for erosion-corrosion wear of the NPP pipelines. Teploenergetika 5: 34-38. [in Russian]

- Chexal VK, Horowitz JS (1995) Chexal-Horowitz Flow-Accelerated Corrosion Model-Parameter and Influences. Current Perspective of Inter. J. Pressure Vessels and Piping: Codes and Standard. Book No. 409768. ASME: 231-243.

- Dooley RB, Chexal VK (2000) Flow-accelerated corrosion of pressure vessels in fossil plants. International Journal of Pressure Vessels and Piping 77: 85-90. https://doi.org/10.1016/S0308-0161(99)00087-3

- Gulina OM, Baranenko VI, Dokukin DA, Yanchenko YuA (2010) Estimation of the erosion-corrosion wear rate and residual life for the NPP piping. Izvestiya vysshykh uchebnykh zavedeniy. Yadernaya energetika 2: 55-63. [in Russian]

- Gulina OM, Frolova OO (2012) Prediction of the NPP equipment lifetime in conditions of erosion-corrosion wear based on an empirical model. Izvestiya vysshykh uchebnykh zavedeniy. Yadernaya energetika 1: 57-65. [in Russian]
- Henzel N, Egan DL (1989) Managing erosion/corrosion with the WATHEC code. Nuclear Engineering International 5: 18-20.

- Horowitz J, Smith D (2013) Recommendation for an Effective Flow-Accelerated Corrosion Program (NSAC-202L-R4). EPRI/3002000563. Technical Report. Palo-Alto, Clf, USA: EPRI, 94 pp.

- Kain V, Roychowhdury S, Mathew T, Bhandakkar A (2008) Flow accelerated corrosion and its control measures for the secondary cycle pipelines in Indian power plants. The Journal of Nuclear Materials 383: 86-91. https://doi.org/10.1016/j.jnucmat.2008.08.024

- Naftal MM, Baranenko VI, Gulina OM (2014) Use of software for calculation of erosion-corrosion wear for the NPP equipment and pipelines. Teploenergetika 6: 1-8. [in Russian]

- Poulson B (1999) Complexities in predicting erosion corrosion. Wear 223-235: 497-504. https://doi.org/10.1016/S00431648(99)00235-5

- Zander A (2012) COMSY - A software tool for aging and plant life management. Proc. of the AREVA NP GmbH, PTCMT-G Third Plant Life Management Conf. May 14-17, Salt Lake City. 